\title{
Remanent magnetic measurements on perpendicular recording materials with compensation for demagnetizing fields
}

\author{
E. O. Samwel \\ MESA Research Institute, University of Twente, P.O. Box 217, 7500 AE Enschede, The Netherlands \\ P. R. Bissell \\ Lancashire Polytechnic, Preston PRI 2TQ United Kingdom \\ J. C. Lodder \\ MESA Research Institute, University of Twente, P.O. Box 217, 7500 AE Enschede, The Netherlands
}

(Received 2 March 1992; accepted for publication 12 October 1992)

\begin{abstract}
Existing techniques for characterization of longitudinal recording media using remanence measurements are extended to perpendicular media, in particular to Alumite, and correction for demagnetizing fields is taken into account. It is found that these techniques have limited value because of the sensitivity of the analysis to the correction factor used. Measurement of the recoil lines is investigated as an alternative method of probing the reversal processes.
\end{abstract}

\section{INTRODUCTION}

During recent years high density recording has become increasingly important as computer hard- and software have developed. Perpendicular recording is one response to this need and very high densities have already been achieved on a laboratory scale. ${ }^{1}$ For example, the (American) Censtor Corporation already produces high density perpendicular hard disks and recently the TEAC company has introduced a new (4 MByte) floppy drive using perpendicular media. ${ }^{2}$ However, the technology is new and there is a need to develop investigation techniques to improve the understanding of the media and to assist further development.

The characterization of longitudinal media is already well developed. Remanence measurements and related procedures, such as "Henkel plots," are widely used to investigate interaction effects. ${ }^{3-5}$ It would seem logical to extend these measurement techniques to perpendicular media. However, the presence of strong demagnetizing fields in the latter makes this difficult and internal fields must be considered during the remanence procedures.

The stimulus for the work reported here was to make measurements on perpendicular media with correction for the demagnetizing field, the generation of remanence curves, switching field distributions, and "Henkel plots" so that a direct comparison with longitudinal media could be made. However, we found that the sensitivity of the results to demagnetization corrections made this type of interpretation inappropriate. This is reported in the article together with the development of an alternative form of measurement which gives some insight into the behavior of the media.

For this study Alumite was chosen as a test material because of its well-known morphology and reversal processes and a series of samples was available with well characterized parameters. In addition, because of its fairly uniform needle structure on a hexagonal lattice, modeling of the demagnetization effects, which is considered in detail elsewhere, ${ }^{6}$ can be undertaken with relative ease.

\section{A. Alumite}

Alumite media, which consists of magnetic columns on a hexagonal matrix, are prepared by electrodeposition of a magnetic material in the pores in aluminum oxide that are formed by the anodic oxidation of aluminum. ${ }^{7}$ A schematic representation of the Alumite morphology is given in Fig. 1. In this figure we see a nearly perfect cylindrical structure grown on initial lobes. By varying the preparation parameters, the column width $\left(D_{p}\right)$ and spacing $\left(D_{c}\right)$ can be controlled and through these, the particle-particle interaction. ${ }^{8}$ The dimensions of the magnetic columns are such that they behave as single domain particles but macroscopically the sample has the properties of a continuous thin film with a perpendicular anisotropy. ${ }^{9}$ In Table I the parameters of the samples reported in this article are given. From scanning electronmicroscopy (SEM) observations it is known that the actual Alumite morphology is very close to the hexagonal array model of Fig. 1 with the exception that not all the pores are completely filled. ${ }^{9}$ Because of this, Alumite is unsuitable for recording purposes at present. In addition, because the exact magnetic volume (and hence the exact magnetization) cannot be determined nondestructively, experimental determination of the demagnetizing field is not possible.

We can consider Alumite as an assembly of columns which have a large aspect ratio (length/diameter). Because the relative separation of the columns is large, the magnetic properties of the film will be dominated by the single particle behavior of the columns together with dipolar interactions. The reversal process in Alumite is known to be generally of a curling-type ${ }^{8}$ which means that the reversal process is initiated by a reversible spin vortex or a flower state at the ends of the columns. This will eventually produce an irreversible switch of the total column moment at some critical field value, which will also be its coercivity. ${ }^{10}$ Thus, we expect the hysteresis loop of a single column to be dominated by irreversible switching producing a square loop with some "rounding" due to reversible processes. Investigation of uncorrected hysteresis loops of Alumite samples indicates sheared loops with a fairly lin- 


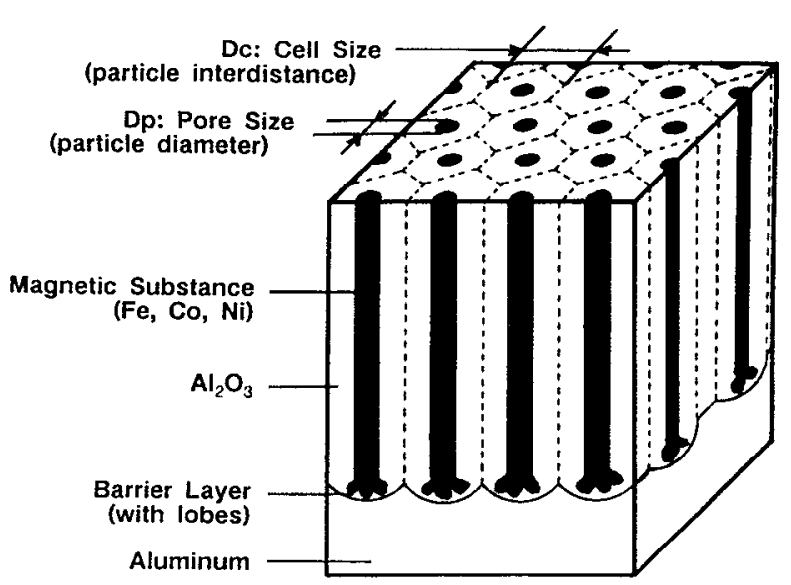

FIG. 1. Alumite morphology.

ear variation of magnetization with applied field around the cocrcivity. We interpret this as indicating that the effect of the dipolar interactions is to generate a "demagnetizationlike" dipolar field which causes the loop to be sheared. In our measurements we have used this assumption in calculating the internal field.

\section{EXPERIMENTAL METHOD AND DISCUSSION}

Various authors ${ }^{11-13}$ have described the correlations between remanence measurements and recording properties in longitudinal media, where the remanence states are compared at the same field value. In perpendicular media we expect these relationships to be different because of the demagnetizing field. In remanence measurements like DCM (dc-demagnetization measurement) and IRM (isothermal remanent magnetization measurement), the magnetic state of a sample which remains when the field is reduced to zero is investigated. Because of the demagnetizing field, the internal field (at zero applied field) will differ significantly from zero in the case of perpendicular media. In order to take full account of the internal behavior and the switching mechanisms, we need to know how the sample behaves as a function of the internal field, in particular at zero internal field. This implies that measurements have to be made as a function of internal rather than applied field, as has been done before by Tomka et al. ${ }^{14}$ on hard materials. In their case, however, the demagnetization factors were much smaller than the sheet demagnetizing factor and intrinsic loops were not square so that correction could be made with reasonable accuracy. However, we have found that in the case of Alumite, because of the large

TABLE I. Alumite sample data.

\begin{tabular}{lcccccc}
\hline Sample & $\begin{array}{c}\text { Filling } \\
\text { material }\end{array}$ & $\begin{array}{c}D_{c} \\
(\mathrm{~nm})\end{array}$ & $\begin{array}{c}D_{p} \\
(\mathrm{~nm})\end{array}$ & $\begin{array}{c}p \\
(\%)\end{array}$ & $\begin{array}{c}H_{c \perp} \\
(\mathrm{kA} / \mathrm{m})\end{array}$ & $\begin{array}{c}M_{s} \\
(\mathrm{kA} / \mathrm{m})\end{array}$ \\
\hline B-1-1 & Fe & 74.5 & 30 & 0.15 & 147 & 240 \\
B-3-3 & $\mathrm{Fe}$ & 74.5 & 45 & 0.33 & 73.4 & 525 \\
Co 7 & Co & 40.5 & 22.5 & 0.28 & 113 & 371 \\
\hline
\end{tabular}

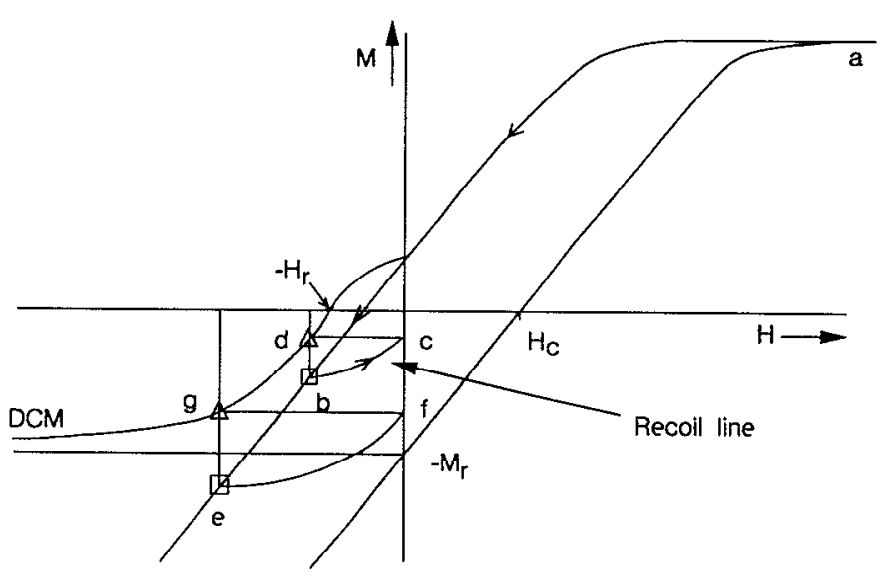

FIG. 2. The DCM measurement procedure, uncorrected for the demagnetizing field (schematic figure).

demagnetizing factor and the intrinsic square hysteresis loops, correction is very sensitive. To make the corrected measurement procedures more clear, let us consider the remanence measurement procedures in terms of both applied field and internal field.

\section{A. Uncorrected DCM measurements}

In the case of longitudinal media, since the demagnetizing field is zero, the internal field and the applied field are always equal. Thus, the remanence states generated by an applied field are always directly related to the field experienced by the particles in the medium. The DCM curve is always measured after saturation of the sample in the positive direction. The DCM measurement procedure against applied field is as follows. Decreasing the field from saturation, the sample state moves from point $a$ to point $b$ on the hysteresis loop in Fig. 2. The field is reduced to zero while the magnetization follows the "recoil line" from point $b$ to point $c$. The magnetization at $c$ is plotted against the field at $b$ to give point $d$ on the DCM curve. The process is repeated for increasing values of negative applied field to generate the full DCM curve. Points $e, f, g$ are another set of points equivalent to $b, c, d$. Returning the system to saturation between the measurement points is not necessary as it has been shown that, within experimental errors, this gives the same results. ${ }^{5}$ The same procedure can be applied to perpendicular media. However, the applied field is no longer that experienced by the particles in the medium and the demagnetizing field now plays an important part. We can still compare the DCM curve and the hysteresis loop by plotting them on the same graph since the difference between them is a measure of the reversible changes as the field relaxes during the field removal. A large separation of the curves indicates large reversible changes.

Another important parameter from the DCM measurement is the remanent coercivity $H_{r}$. This is the value of the field which produces zero remanent magnetization (this state being unaffected by any demagnetizing field). 


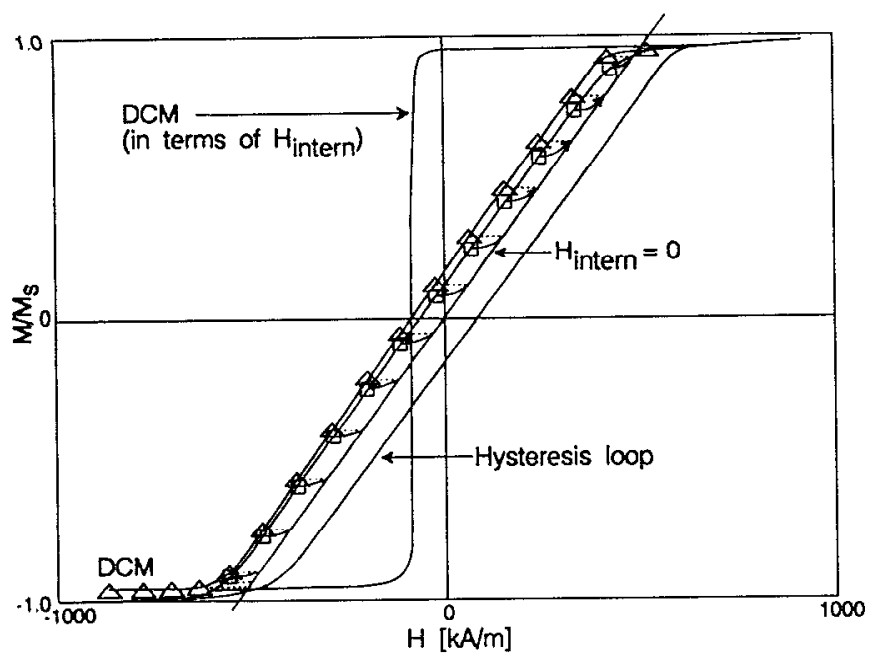

FIG. 3. DCM corrected for the demagnetizing field together with the hysteresis loop and the line where $H_{\text {intern }}=0$. (Alumite sample B-3-3). $\square$ : measurement point on hysteresis loop, $\Delta$ : point on the (uncorrected) DCM curve. The arrows in some of the recoil lines indicate the direction of the recoil.

\section{B. DCM measurements corrected for the demagnetizing field}

We can apply a correction to the applied field in order to obtain a better idea of the process experienced by the particles in the medium. These are two possibilities for this: (1) to correct the field at which the remanence is measured, i.e., remanence at zero internal field; and (2) also to correct the field which generates the remanent state, i.e., to express the whole process in terms of the internal field. In both cases, the remanence is measured at the same applied field, but the DCM curve, when plotted, is expressed against either applied field or internal field. Both methods differ from the uncorrected technique where the remanence is measured at nonzero internal field so that particles will still exhibit some reversible magnetic component. In Fig. 3 we see the results of a corrected DCM measurement on Alumite sample B-3-3 plotted in terms of both internal and applied field. The internal field can be calculated from the applied field using the hysteresis slope at $H_{c}(\beta)$

$$
H_{\text {internal }}=H_{\text {applied }}-\beta^{*} M \text {. }
$$

An important difference between the uncorrected and both corrected measurement procedures is that for the corrected methods, the recoil is always associated with approximately the same field change, whereas without compensation, the field change increases approximately linearly with applied field. This generates a compensated DCM curve which is closer to the hysteresis loop than the uncompensated DCM curve (see Fig. 4).

\section{IRM measurements}

The measurement procedure for the isothermal remanent magnetization curve (IRM) is comparable to the DCM measurement procedure but starting from an initial ac demagnetized state. The sample is magnetized by the

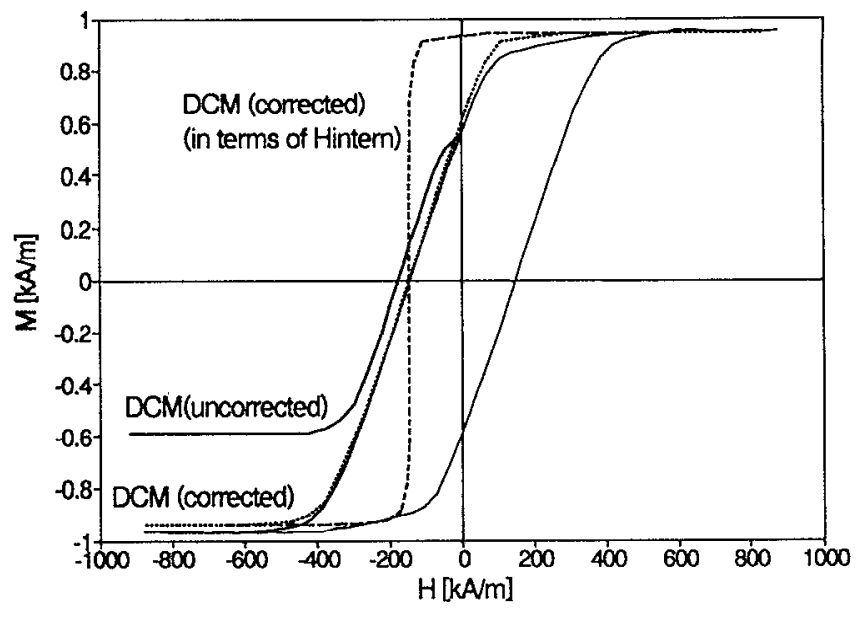

FIG. 4. DCM measurements corrected and uncorrected for the demagnetizing field, plotted against $H_{\text {applied }}$ and $H_{\text {internal, }}$ together with the hysteresis loop. (Alumite sample B-1-1.)

application of increasing dc fields with reduction of the (applied or internal) field at zero. An example of an IRM measurement is given in Fig. 5.

Although IRM and DCM curves are in themselves useful in studying the irreversible magnetization changes in a system, in longitudinal media they are usually analyzed by differentiating to produce the switching field distributions (SFD) or comparison with the Wohlfarth relation ${ }^{4}$ via the Henkel plot. ${ }^{3}$

\section{The Henkel plot}

Comparison of the DCM and IRM remanent states for the same applied field was first made by $\mathrm{Henkel}^{3}$ and gave information about interactions in the magnetic material. ${ }^{4}$ A Henkel plot is derived by plotting $\mathrm{DCM}(\mathrm{H})$ (normalized against the saturation remanence $M_{r}$ ) against the normalized IRM(H) using the field as a connecting factor. It is of vital importance here that IRM and DCM are actually measured in the same quadrant, which means that in

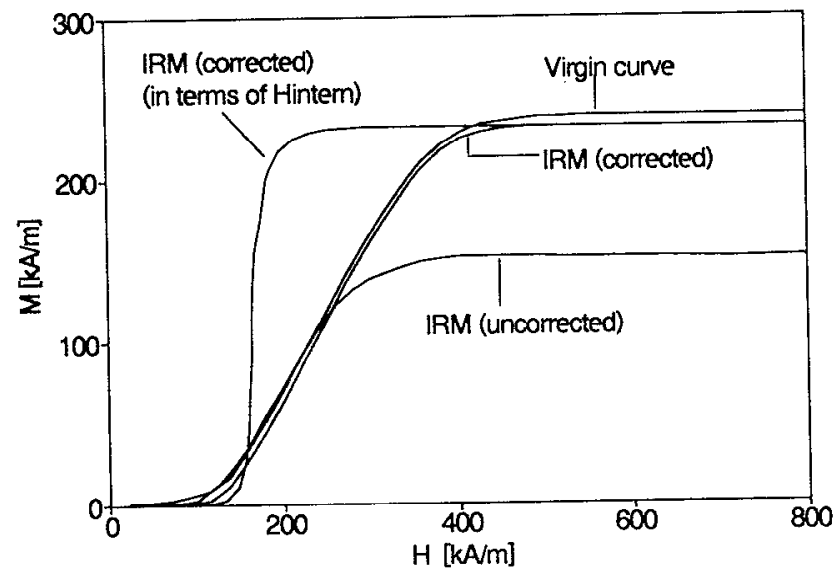

FIG. 5. IRM corrected and uncorrected for the demagnetizing field (Alumite sample B-1-1). For comparison the virgin curve is also plotted. 


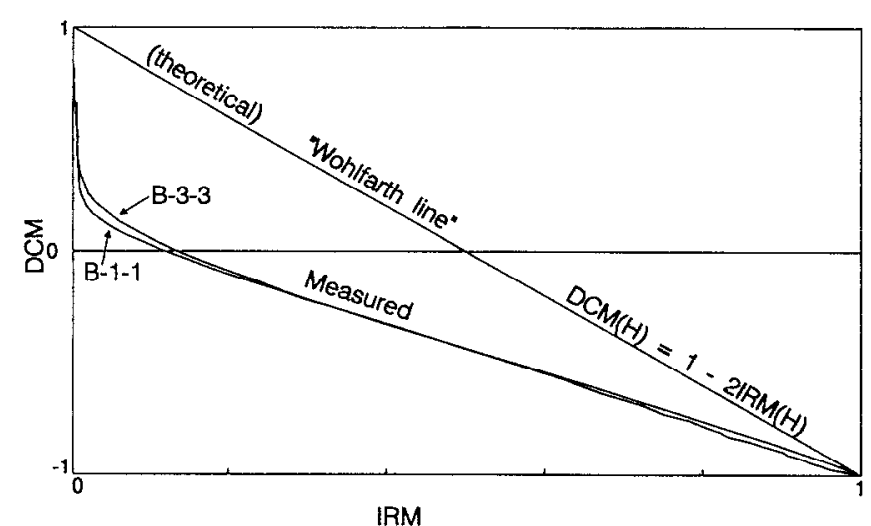

FIG. 6. Henkel plots for Alumite samples B-1-1 and B-3-3 not compensated for the demagnetizing ficld.

this case the DCM should be measured coming from negative saturation, as has been proposed by Lu et al. ${ }^{15}$ This is to prevent that small nonlinearities in the field measurements for positive and negative field values will influence the measurement results. Although Henkel originally plotted the DCM on the horizontal axis, it is now the accepted convention to plot the IRM values on this axis.

In interpretation, Henkel plots are often compared to a line that represents a theoretical system of noninteracting particles. The equation for this line, which was originally derived by Wohlfarth, ${ }^{4}$ is given by $\operatorname{DCM}(H)=1-2$ $\operatorname{IRM}(H)$. A Henkel plot which is below the Wohlfarth line indicates that interactions aid demagnetization. If the plot lies above the line the material opposes demagnetization.

Because of the demagnetizing field the Henkel plots for perpendicular media are far below the Wohlfarth line (see the curves in Fig. 6), which indicates that the interactions aid demagnetization. These interactions, however, are caused by the demagnetizing field and we cannot therefore draw meaningful conclusions from these Henkel curves for perpendicular media.

If we compare the curves in Fig. 6 we see that the demagnetizing field masks most differences. By using the hysteresis loop to calculate the internal field defined by Eq. (1), we can compensate the measurements for the demagnetizing field and the results displayed in Figs. 7 and 8 are derived. If we use the exact value of $\beta$ in Eq. (1), we produce curve $B$. However, experimental errors in the determination of the value for $\beta$ have a dramatic effect on the shape of the curves. To demonstrate this we have plotted curves $C$ and $D$ where the value of $\beta$ has been changed by (plus or minus) $2 \%$, respectively. Since experimental errors in measuring $\beta$ are likely to be of this order the exact shape of the Henkel curve is uncertain.

In Figs. 7 and 8, compensating the curves for the demagnetizing field, the curves move above the Wohlfarth line and the masking effect of the demagnetizing field is reduced. However, as the results depend so strongly on the exact determination of the amount of correction, Henkel measurements have a very limited use for these kinds of media.

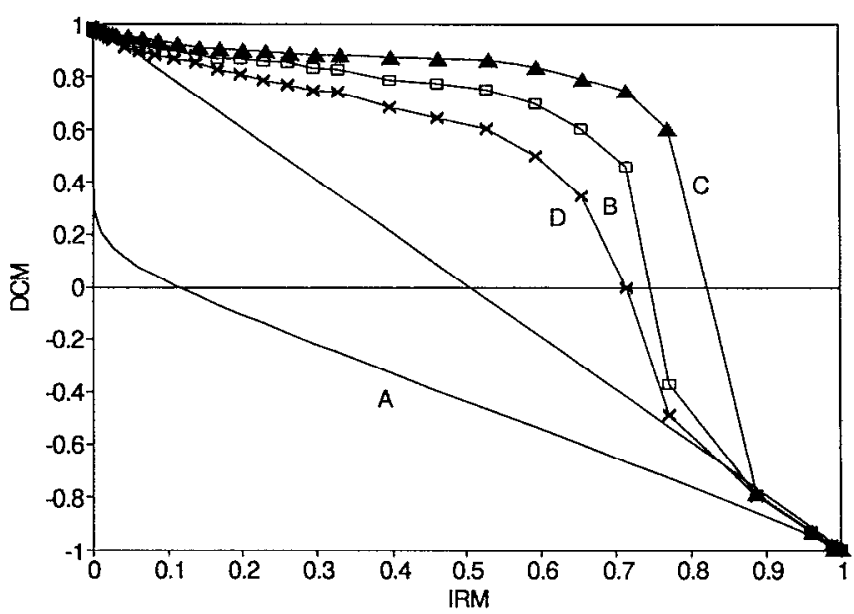

FIG. 7. Henkel plots for Alumite samples B-1-1. (A) Uncompensated, (B) corrected with $\beta$ from the hysteresis loop, (C) $\beta+2 \%$, (D) $\beta-2 \%$.

\section{E. The switching field distribution}

There are many ways of defining a switching field distribution (SFD), either in terms of the hysteresis loop or remanence curves, for longitudinal media. In some cases this is displayed graphically against field whereas other definitions generate a single parameter which characterizes the graph (e.g., the width at half height). The differentials of the IRM and DCM fall in the category of the former and are often used. ${ }^{11}$ In the case of perpendicular media, it should be possible to use this approach, expressing the differentials of the curves either against the applied field or the internal field. In the former, the resulting graph would generally consist of a broad flat topped peak which is again dominated by the demagnetizing field. If the SFD is plotted against the internal field, because of the assumption that the hysteresis loop has an infinite slope at $H_{\mathcal{c}}$, the SFD will be very sharp and the shape will again be sensitive to the choice of the slope $(\beta)$ which is used in the correction [see Eq. (1)] since this determines the slope of the rema-

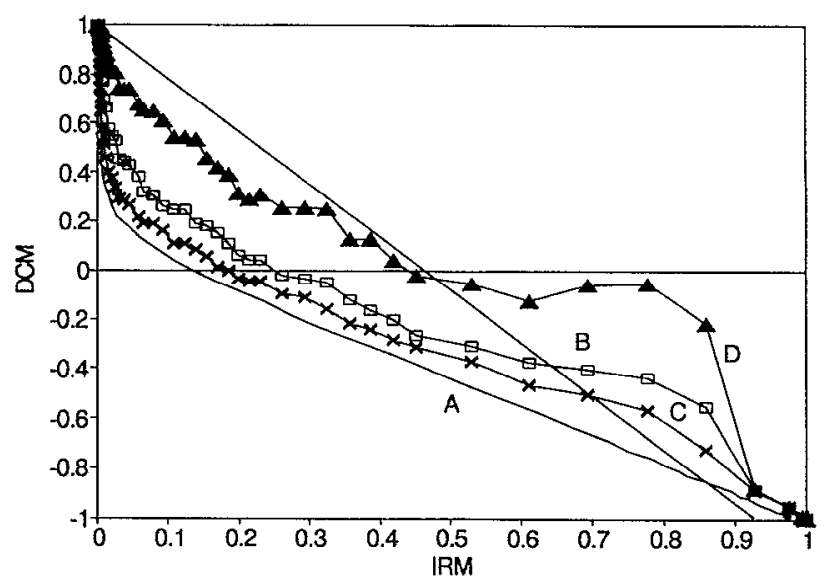

FIG. 8. Henkel plots for Alumite samples B-3-3. (A) Uncompensated, (B) corrected with $\beta$ from the hysteresis loop, (C) $\beta+2 \%$, (D) $\beta-2 \%$. 


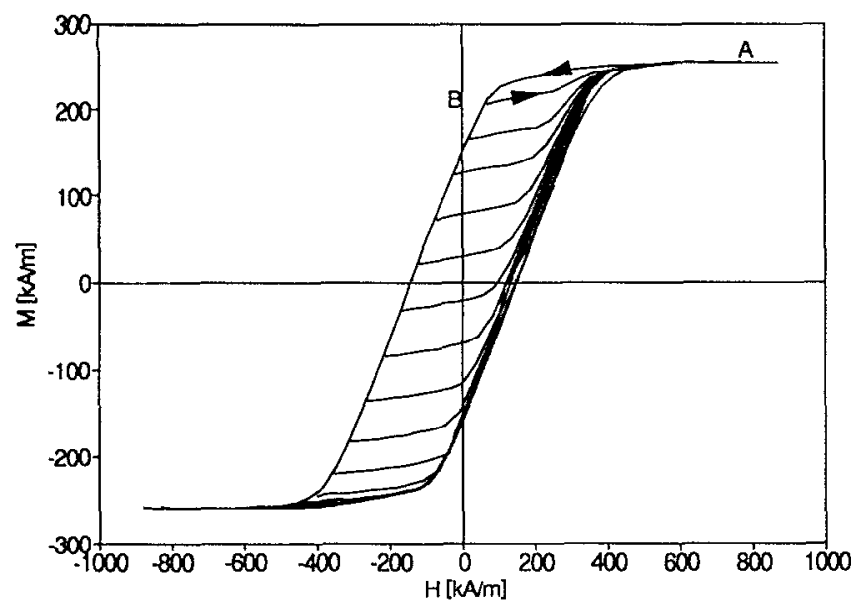

FIG. 9. Recoil lines of iron filled Alumite sample B-1-1. After saturation at $A$, the field is reduced to $B$. The first recoil is measured from $B$ to $A$, along the direction indicated by the arrow.

nence curves. SFDs therefore have little value in characterization of perpendicular media with square intrinsic loops in which strong demagnetizing fields are present.

\section{F. Recoll lines}

In the discussion of the remanence curves, recoil lines have been mentioned as the path that the magnetization follows as the sweep direction is reversed and the field is returned to its starting value. To be more specific we will describe the way that the graph in Fig. 9 has been measured. After saturation (at $A$ ), the applied field is reduced to a certain value $(B)$ and then increased again to saturation. The recoil line is the path that the magnetization follows from $B$ to $A$. This procedure is repeated, each time going to a lower field, until negative saturation is reached. It can be seen that recoil lines and minor loops are closely related. The shape and the initial slope of the recoil lines give useful information about the reversal behavior. The initial slope indicates the ease of reversal of magnetization changes. If the magnetization changes are dominated by irreversible processes, e.g., flux reversal, the slope will be very shallow. However, if the flux changes are dominated by reversible changes, the magnetization changes can be easily reversed and the slope of the recoil line will be relatively steep.

Figures 10 and 11 show two other recoil graphs for Alumite samples which are iron filled and cobalt filled, respectively. For comparison, the recoil lines of a longitudinal sample, a $\gamma-\mathrm{Fe}_{2} \mathrm{O}_{3}$ video tape, are also given (in Fig. 12). From Figs. 9, 10, and 11 we see that even for samples with similar hysteresis loops, recoil lines can have significantly different shapes and slopes. We credit the differences between the recoil lines for the iron-filled samples and the cobalt filled one to the fact that, in the latter, the crystal anisotropy of the individual columns makes an angle with the film plane along which the spins tend to relax (the in-plane anisotropy components will average out so that the film anisotropy direction is perpendicular to the film).

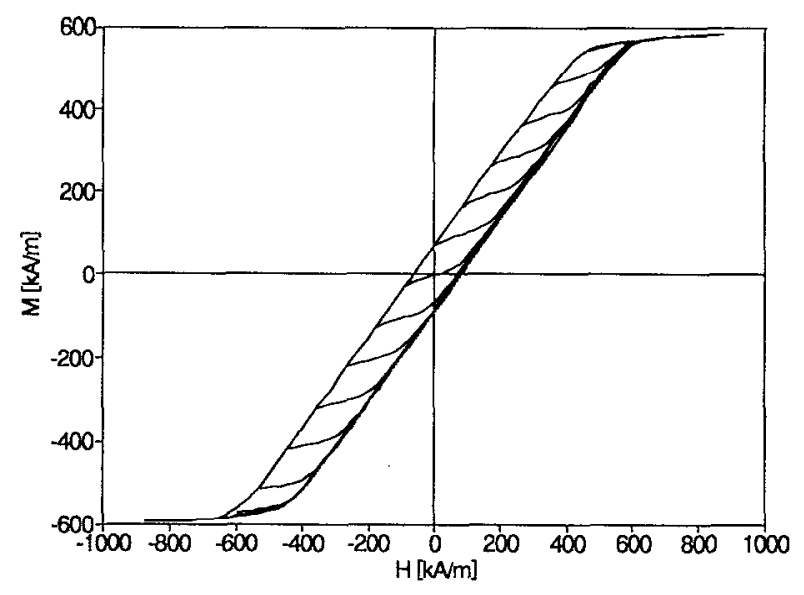

FIG. 10. Recoil lines of iron filled Alumite sample B-3-3.

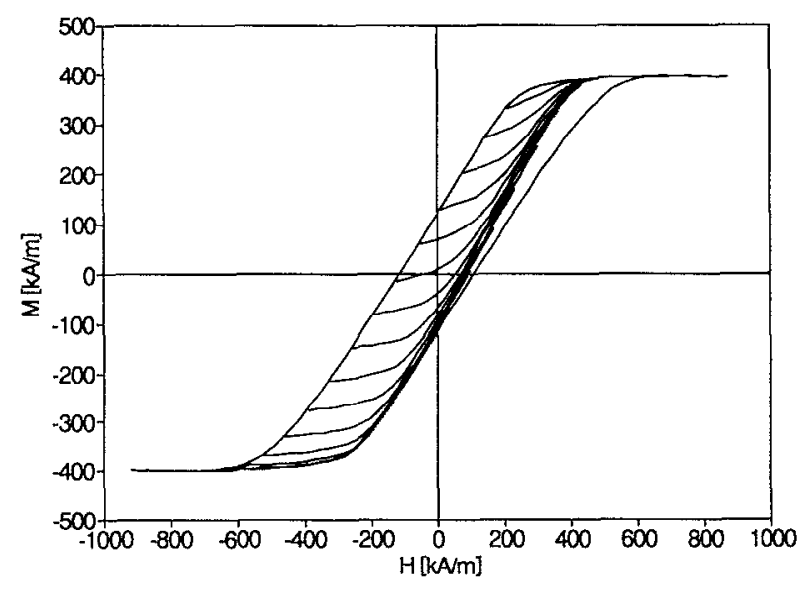

FIG. 11. Recoil lines of cobalt filled Alumite sample Co 7.

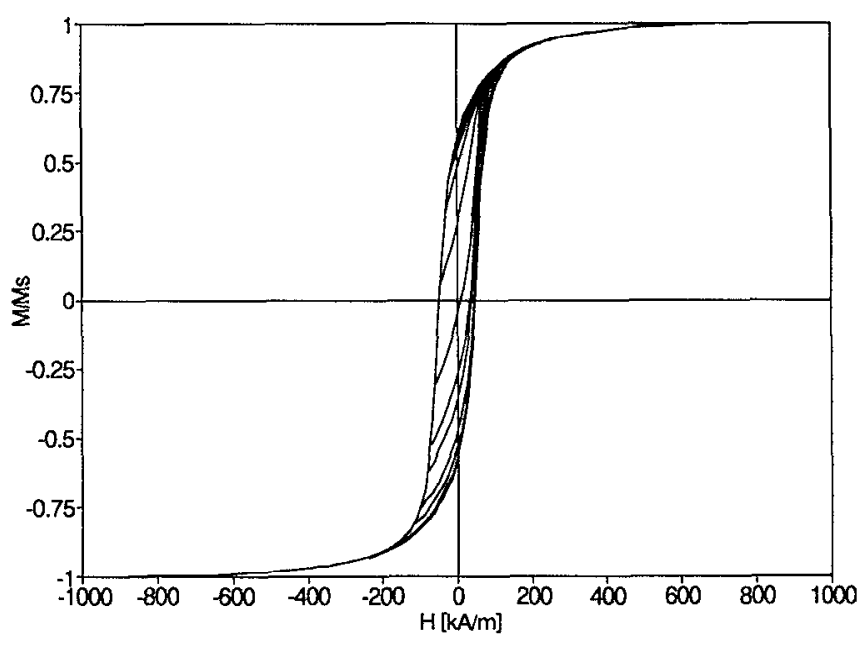

FIG. 12. Recoil lines for $\gamma-\mathrm{Fe}_{2} \mathrm{O}_{3}$ video tape. 


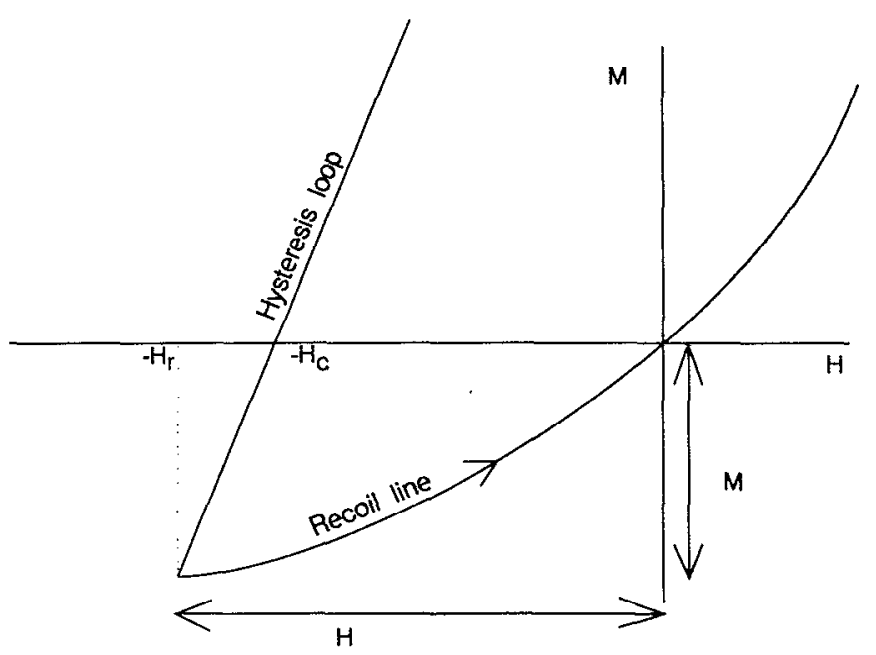

FIG. 13. Calculation of the recoil slope $\rho$.

This effect is not present for the iron filled samples for which only the shape anisotropy is present.

We can characterize the slope of the recoil lines for a system by measuring the change in magnetization along the recoil line which passes through the origin as the field relaxes from its turning value to zero (the turning value will be the remanent coercivity). We can then define a slope parameter equivalent to the mean slope along this recoil line by dividing the magnetization change by the remanent coercivity and normalize this to $M_{s}$. We will call this (recoil shape) parameter $\rho$ (see also Fig. 13).

$$
\rho=\frac{\Delta M}{\Delta H M_{s}} .
$$

From Fig. 13 one can see that $\rho$ is approximately equal to $\left(H_{r}-H_{c}\right) / H_{r}$ (which could be derived from the DCM) if $\beta$ approximates to 1 . Since it is difficult to experimentally generate the recoil line which goes through the origin, we can take the slope of the first recoil line that starts with negative magnetization between its starting point and the point where the internal field is zero. This will give approximately the same value since the recoil lines are almost parallel in that region.

For iron filled Alumite samples, as can be seen from Fig. 14, $\rho$ is related to the sample column diameter. We attribute this to the stiffness of the spins at the ends of the columns due to exchange coupling which will contribute to the curling reversal mechanism. This coupling will be stronger for thinner columns which will oppose reversible behavior. For materials with crystalline anisotropy, such as the cobalt filled Alumite samples, there are other factors influencing the reversible behavior as well, as has been described above, and therefore the simple relationship between the particle size and the amount of reversible behavior (the recoil slope) is not expected. This relationship is being investigated further and also extended to other perpendicular systems.

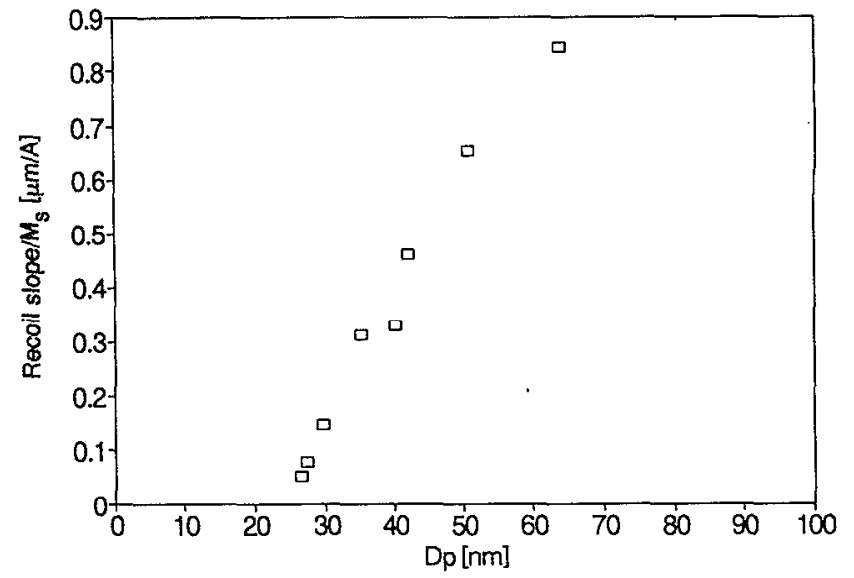

FIG. 14. Normalized recoil slopes $(\rho)$ for different iron filled Alumite samples.

\section{CONCLUSIONS}

We have investigated the hysteretic and remanent properties of alumite, which is a perpendicular medium, as a function of applied and internal field.

Measurements as a function of applied field do not give particularly useful information from the point of view of the recording process since the measurement results are dominated by the demagnetizing field. Measurement of the principal remanence curves against the internal field can be compared via Henkel plots. However, because of their sensitivity to small experimental errors, it is impossible to determine the position of the Henkel curve with any precision.

Switching field distributions derived from uncorrected remanence curves are mainly dominated by the demagnetizing field and SFD as a function of internal field have little meaning because of the sensitivity to errors in determining the exact internal field.

We propose the use of recoil lines as a method of assessing and comparing reversal behavior. The initial slope of the recoil line gives information about the reversible component of the hysteresis loop. The general characteristics of the recoil lines for samples with similar hysteresis loops indicate different reversal behavior and requires further investigation.

The investigation reported in the paper has been limited to Alumite samples and it has been shown that measurements against internal field have a limited use. This work is being extended to look at other perpendicular recording media such as $\mathrm{Co}-\mathrm{Cr}$. However, in some systems where reversal is accompanied by local variations, such as striping out of domains, correction to internal field produces negative slopes which makes the use of Henkel plots, etc., impossible. We therefore feel that the use of recoil lines, which does not depend on correction, provides an additional useful investigative tool for perpendicular media. 


\section{ACKNOWLEDGMENTS}

This work has been carried out within the framework of the CAMST program. One of us (E.S.) is thankful to CAMST for financial support. We would also like to thank Y. Wakui of the Yamaha Research and Development Center for providing the Alumite samples.

${ }^{1}$ S. B. Luitjens, R. W. de Bie, V. Zieren, J. P. C. Bernards, C. P. G. Schrauwen, and H. J. A. Cramer, IEEE Trans. Magn. MAG-24, 2388 (1988).

${ }^{2}$ Computer Info, 5 No. 6 (July 1991) (in Dutch).

${ }^{3}$ O. Henkel, Phys. Status Solidi 7, 919 (1964).

${ }^{4}$ E. P. Wohlfarth, J. Appl. Phys. 29, 595 (1958).

${ }^{5}$ G. W. D. Spratt, P. R. Bissell, R. W. Chantrell, and E. P. Wohlfarth, J. Magn. Magn. Mater. 75, 309 (1988).
${ }^{6}$ E. O. Samwel, P. R. Bissell, and J. C. Lodder, J. Magn. Magn. Mater. 115, 327 (1992).

${ }^{7}$ N. Tsuya, T. Tokushima, M. Shiraki, Y. Umehara, Y. Saito, H. Nakamura, and Y. Harada, IEEE Trans. Magn. MAG-24, 1790 (1988).

${ }^{8}$ G. T. A. Huysmans, J. C. Lodder, and J. Wakui, J. Appl. Phys. 64, 2016 (1988).

${ }^{9}$ E. R. Sterringa, M. Sc. thesis, University of Twente, 1990.

${ }^{10}$ M. E. Schabes, J. Magn. Magn. Mater. 95, 249 (1991).

"G. W. D. Spratt, P. R. Bissell, and R. W. Chantrell, IEEE Trans. Magn. MAG-22, 659 (1986).

${ }^{12}$ D. B. Richards and T. J. Szczech, J. Appl. Phys. 49, 1819 (1978).

${ }^{13}$ E. Köster, H. Jakusch, and U. Kullmann, IEEE Trans. Magn. MAG17, $2250(1981)$.

${ }^{14}$ G. J. Tomka, P. R. Bissell, K. O'Grady, and R. W. Chantrell, IEEE Trans. Magn. MAG-26, 2655 (1990).

${ }^{15}$ M. Lu, Q. Chen, J. H. Judy, and J. M. Sivertsen, J. Magn. Soc. Jpn. 15 S2, 945 (1991). 